\title{
Research Paper: Tracking the Transplanted Neurosphere in Retinal Pigment Epithelium Degeneration Model
}

\author{
Hamid Aboutaleb Kadkhodaeian ${ }^{1,2}$ (D), Amir Salati ${ }^{13^{*}}$ (D), Mojtaba Ansari ${ }^{4}$ (D), Vajihe Taghdiri Nooshabadi ${ }^{3}$ (D) \\ 1. Nervous System Stem Cells Research Center, Semnan University of Medical Sciences, Semnan, Iran. \\ 2. Department of Anatomical Sciences, School of Medicine, Semnan University of Medical Sciences, Semnan, Iran \\ 3. Department of Tissue Engineering and Applied Cell Sciences, School of Medicine, Semnan University of Medical Sciences, Semnan, Iran. \\ 4. Department of Biomedical Engineering, University of Meybod, Meybod, Yazd, Iran.
}

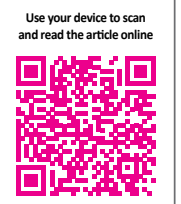

Citation: Kadkhodaeian, H. A., Salati, A., Ansari, M., \& Taghdiri Nooshabadi, V. (2021). Tracking the Transplanted Neurosphere in Retinal Pigment Epithelium Degeneration Model. Basic and Clinical Neuroscience, 12(4), 523-532. http://dx.doi. org/10.32598/bcn.2021.12.4.2230.1

http://dx.doi.org/10.32598/bcn.2021.12.4.2230.1

\section{Article info:}

Received: 26 Nov 2019

First Revision: 30 Sep 2020

Accepted: 02 Jun 2021

Available Online: $01 \mathrm{Jul} 2021$

Keywords:

Neurosphere cells, Bone marrow stromal stem cells, Sodium iodate, Age-Related macular degeneration

\section{A B S T RA C T}

Introduction: Retinal Pigment Epithelium (RPE) layer deterioration is a leading cause of AgeRelated Macular Degeneration (AMD), i.e., the most significant reason for irreversible blindness. The present study aimed to track the Neurosphere-Derived (NS) from Bone Marrow Stromal Stem Cells (BMSCs) grafted into the sub-retinal space (destruction of the RPE layer by sodium iodate).

Methods: RPE degeneration model was performed using the injection of $5 \%$ sodium iodate performed in the retro-orbital sinus of Wistar rats. BMSCs were extracted from the examined rat femur and induced into NS, using EGF, bFGF, and B27. BrdU-NS labeled cells were transplanted into the sub-retinal space. For detecting BMSCs and NS markers, immunocytochemistry was performed. Moreover, immunohistochemical was conducted for tracking the transplanted cells in the RPE and sensory retina.

Results: The immunocytochemistry of BMSCs cells displayed the expression of mesenchymal stem cells markers (CD90; 99\% \pm 1$)$, CD166 $(98 \% \pm 2)$, CD44 $(99 \% \pm 1)$. Additionally, the expression of neural lineage markers in NS, such as SOX2, OCT4, Nanog, Nestin, and Neurofilaments $(68,160,200)$ revealed the differentiation from BMSCs. Tracking BrdU-NS labeled suggested these aggregations in most layers of the retina.

Conclusion: Our study data indicated that BMSCs derived neurosphere had the potential to migrate in injured retinal and integrate into the neurosensory retina. These data can be useful in finding safe cells for replacement therapy in AMD.

\section{* Corresponding Author:}

Amir Salati, PhD.

Address: Nervous System Stem Cells Research Center, Semnan University of Medical Sciences, Semnan, Iran.

Tel: +98 (912) 8683983

E-mail: amirsalati@yahoo.com 


\section{Highlights}

- Differentiation of bone marrow stromal stem cells into Neurosphere.

- Migration of BMSC derived Neurosphere into the neurosensory retina.

- Survival, migration, and integration of BMSC derived Neurosphere in Subretinal space.

\section{Plain Language Summary}

Age-Related Macular Degeneration (AMD) is a common condition leading to blindness. Different types of stem cells are used to replace lost cells during this disease. Among them, neural lineages stem cells are the most convenient cells because of their origin. These cells turn to retinal pigment epithelium and neurosensory retina as in embryological time. It seems that transplantation of neurosphere, a neural linage stem cell, could be a promising procedure to study the restoration mechanism in AMD.

\section{Introduction}

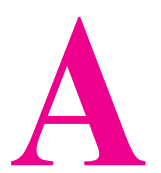

ge-Related Macular Degeneration (AMD) is the most prominent reason for visual disorders among the elderly in advanced communities; it occurs in populations over the age of 50 years (Paulus \& de Jong, 2006; Weed \& Mills, 2017). Commonly, the risk factors for this condition are smoking cigarettes, diet, gender, and family history (Ambati, Ambati, Yoo, Ianchulev, \& Adamis, 2003; Chen et al., 2016). The macula is a highly sensitive part of the retina and does not exist in rodents (Franco et al., 2009; Machalińska et al., 2010). Therefore, animal models are diminished for this disorder. There is no ideal model for investigating AMD; however, models with a pathology like human disease are proper (Enzmann et al., 2006; Pennesi, Neuringer, \& Courtney, 2012). Numerous pathological appearances are similar in human and animal models. For example, the formation of drusen, thickening of Bruch membrane, the degeneration of the retina, choroidal Neo-Vascularization, and the degeneration of PRE layer in different parts.

There is no pharmacotherapy for patients with AMD. Macular translocation and RPE transplantation surgeries decrease visual loss and improve vision in some patients (Stanga et al., 2002). Studies demonstrated that normal RPE can ease the survival of photoreceptors; it supports the potential for use of stem cells and RPE derivatives, like resources for AMD treatment (Song et al., 2018). Using the ability of stem cells to replace and repair damaged tissue in AMD and Retinitis Pigmentosa (RP) was suggested. The therapies stem cell-based can either replace the Retinal Pigment Epithelium (RPE), or the neurosensory retina (Lee \& MacLaren, 2011). Several studies assessed the potential and effect of stem cells and RPE transplantation to therapy of AMD.

Replacing the injured cells of the retina with stem cells, i.e., renewables and differentiable is proposed as a strategy (Parameswaran \& Krishnakumar, 2017; Park et al., 2017; Schwartz, Nagiel, \& Lanza, 2017). It has done multiple considerations to identify the best of the cell for transplantation in the retina. Likewise, the ordering of cells provides a convenient space for sub-retinal injection. Different cells were manipulated to generate PRE cells and photoreceptors (Mead et al., 2015; Park et al., 2017; Trounson \& McDonald, 2015; Weed \& Mills, 2017).

Fully-Differentiated cells may be useful for transplants to replace RPE or photoreceptors cells in the retina. However, fully differentiated cells may be less likely to survive and migrate into the host tissue. Neural stem cells can differentiate into neural linage cells, such as a neuron, astrocytes, and oligodendrocytes. Therefore, neurosphere and neural stem cells seem to be more useful than fully differentiated cells for retinal diseases (Yu et al., 2008) like AMD. There is a limitation to generate the neurosphere from the brain donors; however, mesenchymal stem cells (BMSCs, ADSCs, etc.) (Li, Liao, Gong, Yuan, \& Tan, 2009) can differentiate into the cell the express neural and glial markers, advising these cells could be used for retinal degenerative diseases, like AMD (Ferroni et al., 2012; Fu et al., 2008; Kaka, Tiraihi, Arab, \& Azizzadeh, 2009; Liu et al., 2013; Wu et al., 2003; Yang et al., 2010). 
Retinal and RPE layers originate from the neural tube during the embryonic period; thus, we hypothesized that BMSCs-derived neurosphere can migrate and differentiate into RPE/retinal cells. Therefore, this study investigated survival and migration into the RPE layer and neurosensory retinal.

\section{Methods}

All of the procedures on the Wistar male rats (Razi institute, Iran, Tehran), including euthanasia, were performed per the Association for Vision Research and Ophthalmology (ARVO) statement for the use of animals in ophthalmic and vision research and with the Helsinki Declaration of 1975, as revised in 2013. Twenty rats were housed with a 12:12h light/dark cycle. The animals were randomly divided into the experimental (received cells into sub-retinal space, $n=10$ ) and control (without cell injection, $\mathrm{n}=10$ ) groups.

RPE degeneration was performed with some modifications (Aboutaleb et al., 2017). Briefly, Sodium iodate (Sigma, St. Louis, MO, USA) was dissolved in Phosphate-Buffered Saline (PBS) to $2.5 \mu \mathrm{g} / \mathrm{mL}$ and stored at $4^{\circ} \mathrm{C}$. The explored animals were anesthetized and pupillary dilation was achieved with $0.5 \%$ phenylephrine eye drop and $0.5 \%$ tropicamide (Santen, Osaka, Japan). $\mathrm{NaIO}_{3}$ was injected using an insulin needle at $45^{\circ}$ to the nose into the retro-orbital venous plexus, after complete dilatation of the pupil.

For performing cell culture, the BMSCs were prepared from the bone marrow of the Wistar male rats (6- to 8-week-olds) according to the method previously described (Darabi et al., 2013). The bone marrow was obtained from the tibias and femurs with a $5 \mathrm{~mL}$ syringe containing Dulbecco's modified Eagle's medium (DMEM-GIBCO-BRL, Eggenstein, Germany). The freshly isolated cells were resuspended in a DMEM supplement containing 10\% fetal bovine serum (FBS: GIBCO-BRL, Eggenstein, Germany), $100 \mathrm{U} / \mathrm{mL}$ penicillin $\mathrm{G}$, and $100 \mathrm{mg} / \mathrm{mL}$ streptomycin sulfate (penicillin/streptomycin: GIBCO-BRL, Eggenstein, Germany). Next, they were added to T25 flasks (Nunc, Wiesbaden, Germany). The non-adherent cells were removed after $24 \mathrm{~h}$. The adherent ones were used as the primary culture and were used after the third passage. Furthermore, the characterization of the BMSC was conducted according to a previous study (Darabi et al., 2013). The BMSCs were characterized using the primary antibodies (Table 1). This method was performed as mentioned in the immunocytochemistry procedure.
For neurosphere generation and labeling, the generation of neurospheres from the BMSC was conducted as previously explained (Darabi et al., 2013). The harvested BMSCs $\left(104\right.$ cells $\left./ \mathrm{cm}^{2}\right)$ at the third passage were seeded into 6 wells in neurosphere production medium consisting of DMEM/F12 supplemented by EGF (20 ng/ $\mathrm{mL}$ ) (Sigma-Aldrich, Germany), 2\% B27 (Invitrogen, Karlsruhe, Germany) and $20 \mathrm{ng} / \mathrm{mL}$ of basic fibroblast growth factor (bFGF; Chemicon, Hofheim, Germany). The characterization of the neurospheres was performed by the primary antibodies (Table 2). For labeling, added BrdU to the culture medium for a concentration of $7.81 \mu \mathrm{L} / 2.5 \mathrm{~mL}$ medium and incubated at $37^{\circ} \mathrm{C}$ for $72 \mathrm{~h}$. The technique was carried out for the characterization of neurosphere cells and BrdU labeling detection as mentioned in the immunocytochemistry section.

The transplantation of BMSCs into the subretinal space was performed as follows: Sub-retinal space injection was conducted according to a previous study (Aboutaleb et al., 2017). Briefly, the examined rats were injected with $40 \mathrm{mg} / \mathrm{kg}$ of sodium iodate (Sigma) into the retro-orbital sinus. Besides, the study animals were prepared for cell transplantation 30 days later. Rats were anesthetized with topical $1 \%$ proparacaine eye drops (Santen) and intraperitoneal 2\% pentobarbital (40 mg/ $\mathrm{kg}$; Santen, Osaka, Japan). Pupillary dilation was prepared with $0.5 \%$ phenylephrine eye drops and $0.5 \%$ tropicamide (Santen). Then, the anesthetized animals were placed in lateral recumbency under Zeiss dissecting microscope and positioned by a holding hand. The rat fundus was visualized with the application of a drop of $2.5 \%$ methylcellulose to the eye. The cornea was carefully punctured nasally almost $0.5 \mathrm{~mm}$ to $1 \mathrm{~mm}$ medial to the dilated pupillary margin by a 28-gauge hypodermic needle (Becton Dickinson \& Company, Franklin Lakes, NJ, USA). The needle with the bevel up was advanced through the full thickness of the cornea into the anterior chamber parallel to the anterior lens face. The half of the bevel was pushed through the cornea, producing a hole large enough to insert the 33-gauge blunt needle (Hamilton Company, Reno, NV, USA). The blunt needle tip was inserted through the corneal puncture and advanced into the anterior chamber, avoiding trauma to the iris and lens. Consequently, the needle shaft was aimed slightly nasally toward the posterior chamber with the iris lateral and lens medial. The lens was displaced medially as the needle was advanced toward the appropriate location of injection. Slight resistance to the movement of the needle indicated the penetration of the retina and entrance into the subretinal matrix. Subsequently, the syringe was held in place, while an assistant pushed the BMSCs suspension $(10-\mu \mathrm{L}$ with a concentration of 107 cells $/ \mathrm{mL})$ 
slowly over almost 30 seconds, injecting the contents of the syringe into the subretinal matrix and creating a visible retinal detachment. After subretinal delivery, the needle was gently withdrawn (Figure 1).

The immunocytochemical study was performed on the cells or neurospheres, i.e., fixed into $4 \%$ paraformaldehyde for $15 \mathrm{~min}$, washed, permeated using triton $\mathrm{x}-100(0.3 \%)$, blocked in goat serum at $37^{\circ} \mathrm{C}$ for one $\mathrm{h}$, labeled with various primary antibodies (Tables $1 \& 2$ ) at $4^{\circ} \mathrm{C}$ for $24 \mathrm{~h}$ (the negative $\&$ positive controls were simultaneously immunostained); incubated with secondary antibodies conjugated with FITC at $37^{\circ} \mathrm{C}$ for one $\mathrm{h}$, and counterstained with propidium iodide $(20 \mu \mathrm{g} / \mathrm{mL}$, at room temperature for $5 \mathrm{~min}$ ), respectively. The cells were visualized by a fluorescent microscope (Olympus 1x71, Olympus, Tokyo, Japan). The estimation of the immunoreactivity percentages of the induced cells was conducted as previously described (Naghdi, Tiraihi, Mesbah-Namin, \& Arabkheradmand, 2009); several images were selected randomly and the number of immunoreactivity cells for a given antibody was divided by the total number of the cells.

Immunohistochemistry investigation was performed as follows: At the termination of experimental procedures and where histological post-processing was required, rats were terminally anesthetized by xylazine $(4 \mathrm{mg} / \mathrm{kg})$ and ketamine $(40 \mathrm{mg} / \mathrm{kg})$ IP injection. The eyes were enucleated and placed in a fixative at $4^{\circ} \mathrm{C}$ for $2 \mathrm{~h}$. The anterior chamber, lens, and neural retina were removed for producing eyecups that the RPE was exposed. To access whole-mounts, 4 equidistant cuts were made in the eyecups. Auto-Fluorescence micrographs were produced using an excitation wavelength of $>498 \mathrm{~nm}$ of the RPE sheet before immunohistochemistry. For cross-section, enucleated eyes were fixed with PAF $4 \%$ overnight and hydrated, and then blocked with paraffin. Furthermore, the 5-7 $\mu \mathrm{m}$ sagittal section was done. For BrdU detection, section hydrate in routine process and after antigen retrieval with trypsin, sections incubated with primary antiBrdU $2 \mathrm{~h}$ at room temperature (humidity chamber); then, they were incubated with secondary anti-BrdU conjugated with FITC $1 \mathrm{~h}$ in RT. After the rinse, the sections were counterstained with 4'6-diamindino-2-phenylindole dihydrochloride (DAPI, Sigma-Aldrich, Pool Dorset, UK) and observed under the fluorescent microscope.

The obtained data were expressed as mean $\pm \mathrm{SE}$ values for the experimental rats and controls. The collected data were tested for normality using the Kolmogorov-Smirnov test, suggesting no departure from the normal distribution. Then, the achieved data were analyzed by Independent Samples t-test in SPSS v. 15 . $\mathrm{P}<0.05$ was considered significant.

\section{Results}

Bone marrow stem cells were extracted from the bone marrow tissue of rats (weight 200-250 gr). These cells were cultured to become uniform in shape (four passage). These cells had a diverse appearance and fibroblastic shape, distinct from other cells (Figure 2 A-E). An examination of immunocytochemistry revealed that these cells express fibronectin markers and surface markers of CD90, CD166, and CD44; however, the surface markers of CD34 (representing hematopoietic stem cells), Nestin (representing neural cells), and GFAP (representing glial cells) does not express.

To differentiate the bone marrow stem cells into neurosphere cells, the method of Lijuan Fu et al. with a slight change was used Kadkhodaeian, Salati, \& Lashay (2019). BMSCs cells were separated from the flask after passage 4

Table 1. The list of primary antibodies for the immunocytochemistry of BMSCs

\begin{tabular}{ccccc}
\hline Primary antibody & Host & Titer & Cell & Supplier \\
\hline Anti- Fibronectin & Mouse & $1: 500$ & BMSCs & Abcam, Cambridge, UK \\
Anti-CD90 & Mouse & $1: 500$ & Undifferentiated cell & Abcam, Cambridge, UK \\
Anti-CD166 & Rabbit & $1: 100$ & Stromal cell & Abcam, Cambridge, UK \\
Anti-CD44 & Rabbit & $1: 100$ & Stromal cell & Abcam, Cambridge, UK \\
Anti-CD34 & Mouse & $1: 100$ & Hematopoietic cell & Abcam, Cambridge, UK \\
Anti-Nestin & Mouse & $1: 50$ & Neural stem cell & Abcam, Cambridge, UK \\
Anti- GFAP & Rabbit & $1: 400$ & Neural stem cell & Abcam, Cambridge, UK \\
\hline
\end{tabular}


Table 2. Primary antibodies for the characterization of neurospheres

\begin{tabular}{cllll}
\hline Primary Antibody & Host & Titer & Cell & Supplier \\
\hline Anti-SOX2 & Rabbit & $1: 300$ & Neurosphere & Abcam, Cambridge, UK \\
Anti-OCT4 & Mouse & $1: 300$ & Neurosphere & Abcam, Cambridge, UK \\
Anti-Nanog & Mouse & $1: 300$ & Neurosphere & Abcam, Cambridge, UK \\
Anti-NF68 & Mouse & $1: 300$ & Neurosphere & Abcam, Cambridge, UK \\
Anti-NF160 & Mouse & $1: 300$ & Neurosphere & Abcam, Cambridge, UK \\
Anti-NF200 & Mouse & $1: 300$ & Neurosphere & Abcam, Cambridge, UK \\
Anti-nestin & Mouse & $1: 300$ & Neurosphere & Abcam, Cambridge, UK \\
\hline
\end{tabular}

NEUR SCIENCE

in a culture medium and centrifuged at $1500 \mathrm{rpm}$ for $3 \mathrm{~min}$ The cellular pellet with a neurosphere differentiating medium, containing DMEM/F12, plus $20 \mathrm{ng} / \mathrm{ml}$, EGF, bFGF, and B27, were suspended and divided into a 6 wells plate. After about an hour, the cells began to aggregate and new cellular assemblies were created. One day after, the cell aggregate was detected in the medium (Figure $3 \mathrm{~A}, \mathrm{~B}$ ). Cell aggregates were placed in the same culture medium for seven days and their medium changed every two days. Immunocytochemistry was used to confirm the neurospheres cells. The expression of nuclear markers revealed the stemness of these cells, for example, SOX2, Nanog, and Oct 4 (Figure $3 \mathrm{C}-\mathrm{E}$ ). Additionally, the expression of cytoplasmic markers, neurofilaments 68, 160, and 200 (Figure 3 A-C), was observed.

Seven days after the transplantation of the neurosphere cells into sub-retinal space, the rat's eyes were removed; after the preparation of the paraffin mold, sections were made in the thickness of $7.5 \mu \mathrm{m}$. The slides were checked for tracking cells labeled with a BrdU nuclear marker in the RPE layer as well as the neural retina. The sections were stained with DAPI nuclear marker where the transplanted cells, as well as the host cells, can be well-characterized. At the injection site (Figure 4-D), the accumulation of neurosphere cells was observed in binding to the RPE and retina layer. A number of these cells were observed in the sub-retinal space of the retina; however, most of them were moved into the RPE layer and the retina. In some places, BrdU positive cells were observed into the choriocapillaris layer (Figure 4C and F). The injected neurospheres were identified in the clamp and single-cell form in the sections (Figure 4B, C). Seven days after cell transplantation, neurosphere BrdU positive cells seem to have survived into the sub-retinal space and migrate to the RPE layer.

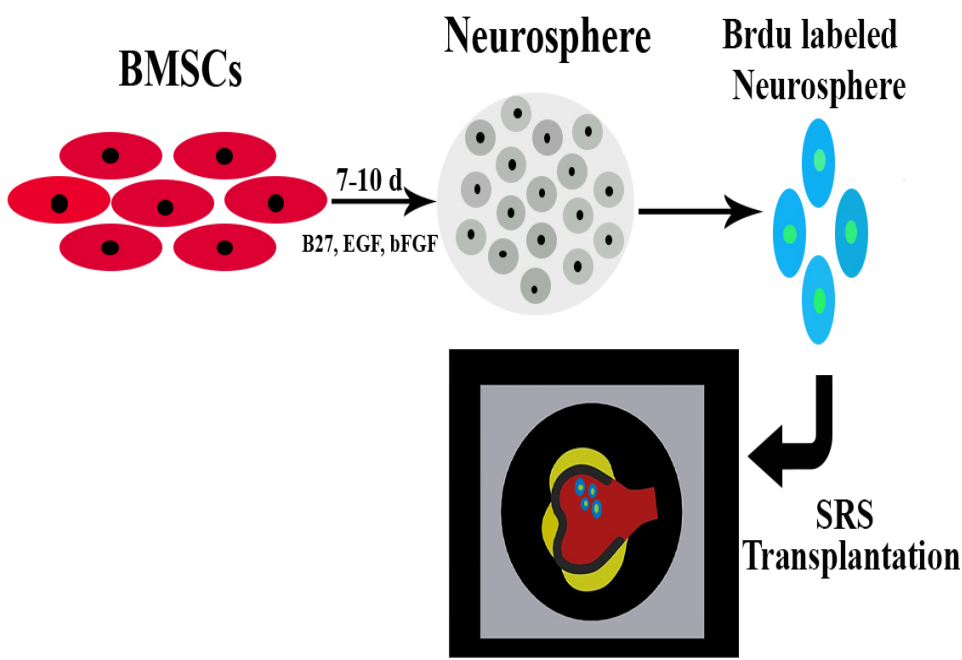

Figure 1. The schematic derivation of neurosphere BMSCs and transplantation into subretinal space 

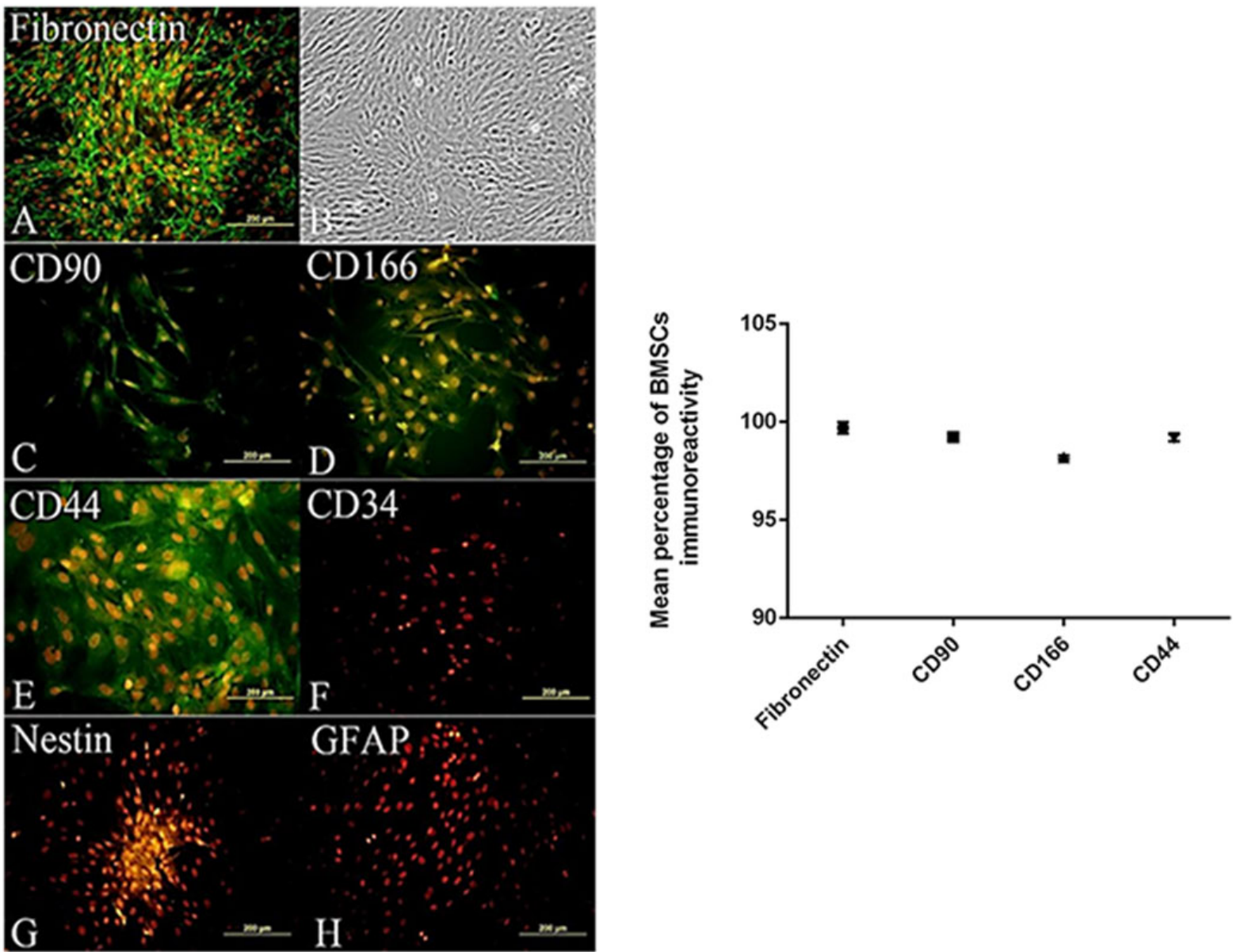

Figure 2. The isolation and culture of stem cells derived from bone marrow tissue in rats in passage 4

A-H: The immunocytochemistry of bone marrow stem cells with Fibronectin; B: Represents the contrast phase image of the bone marrow stem cells.

The images represent markers CD90, CD166, CD44, CD34, Nestin, GFAP, respectively. The green fluorescence light is related to antibodies conjugated to FITC. The nucleus was counterstained with propidium iodide. Magnification: $200 \mu \mathrm{m}$ for A-H.

Figure 4 shows the migration of neurosphere cells labeled with a BrdU in the retinal layers. As shown in Figure 4-C, and D, neurosphere cells were homed in the RPE layer and the retinal layers (Figure 4-B, D, and F). In the sensory retina, the obtained data suggested that the BrdU positive cells are located in the inner nuclear layer and ganglionic layer (Figure 4-B, and F). The number of cells in the inner nuclear layer was more than the outer nuclear layer. As before mentioned, BrdU positive neurosphere cells were observed as a single cell and aggregate (Figure 4-B). The relevant results highlighted that 7 days after cell transplantation, the neurospheres could survive in the location and migrate to the sensory retina. In this study, the migration of neurospheres was not detected in the vitreous after injection of cells in the sub-retinal space of the retina. The results of this section indicate that 7 days after transplantation, the BrdU positive neurospheres cells survived into the sub-retinal space after injection and migrated to the RPE layer and the neurosensory retina. The migration occurred in the pigmentation layer and the retina layers, including the inner nuclear layer and the ganglionic layer.

\section{Discussion}

Systematic delivery of sodium iodate $\left(\mathrm{NaIO}_{3}\right)$, a stable oxidizing agent, was proven to be an effective way to induce retinal degeneration associated with the regional loss of Retinal Pigment Epithelium (RPE) recapitulating some of the morphological features of geographic atrophy. $\mathrm{NaIO}_{3}$ retinal toxicity was demonstrated in many different mammalian species, including sheep, rabbits, rats, and mice. $\mathrm{NaIO}_{3}$ is thought to directly affect the RPE cells with secondary effects on photoreceptors. Moreover, the 


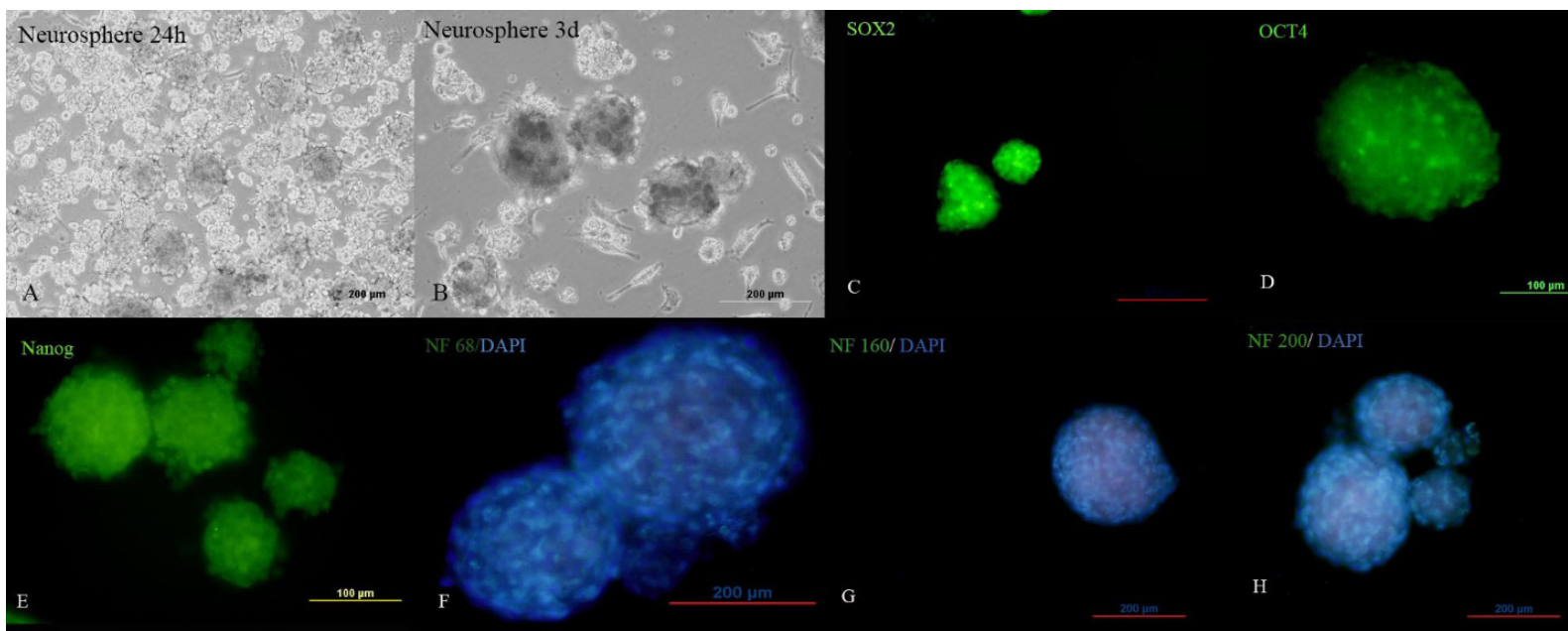

NEUR SCIENCE

Figure 3. The morphological and immunocytochemistry images of neurospheres derived from bone marrow stromal stem cells

A, B: Represented the neurosphere morphology in one and three days after differentiation; C-E: Represented the SOX2, OCT4, and Nanog nuclear markers immunocytochemistry; F-H: Represented the neurofilaments 68, 160, and 200 immunocytochemistry conjugated with FITC and nuclear counterstained with DAPI. Magnification: $100 \mu \mathrm{m}$ for C-E, and $200 \mu \mathrm{m}$ for A, B, F-H.

choriocapillaris induces the production of reactive oxygen species contributing to damages in RPE cells. Other effects of $\mathrm{NaIO}_{3}$ on RPE cells include the inhibition of enzyme activity (e.g., triose phosphate dehydrogenase, lactate dehydrogenase) in RPE cells, disruption of the blood-retina barrier, and increased conversion of glycine to potentially toxic glyoxylate by melanin (Franco et al., 2009; Jiang, Zhang, \& Chiou, 2009; Koh et al., 2019; Zieger \& Punzo, 2016).

The present study revealed that transplanted of neurosphere derived from BMSCs in AMD model, migrated and incorporated into the RPE layer. We demonstrated
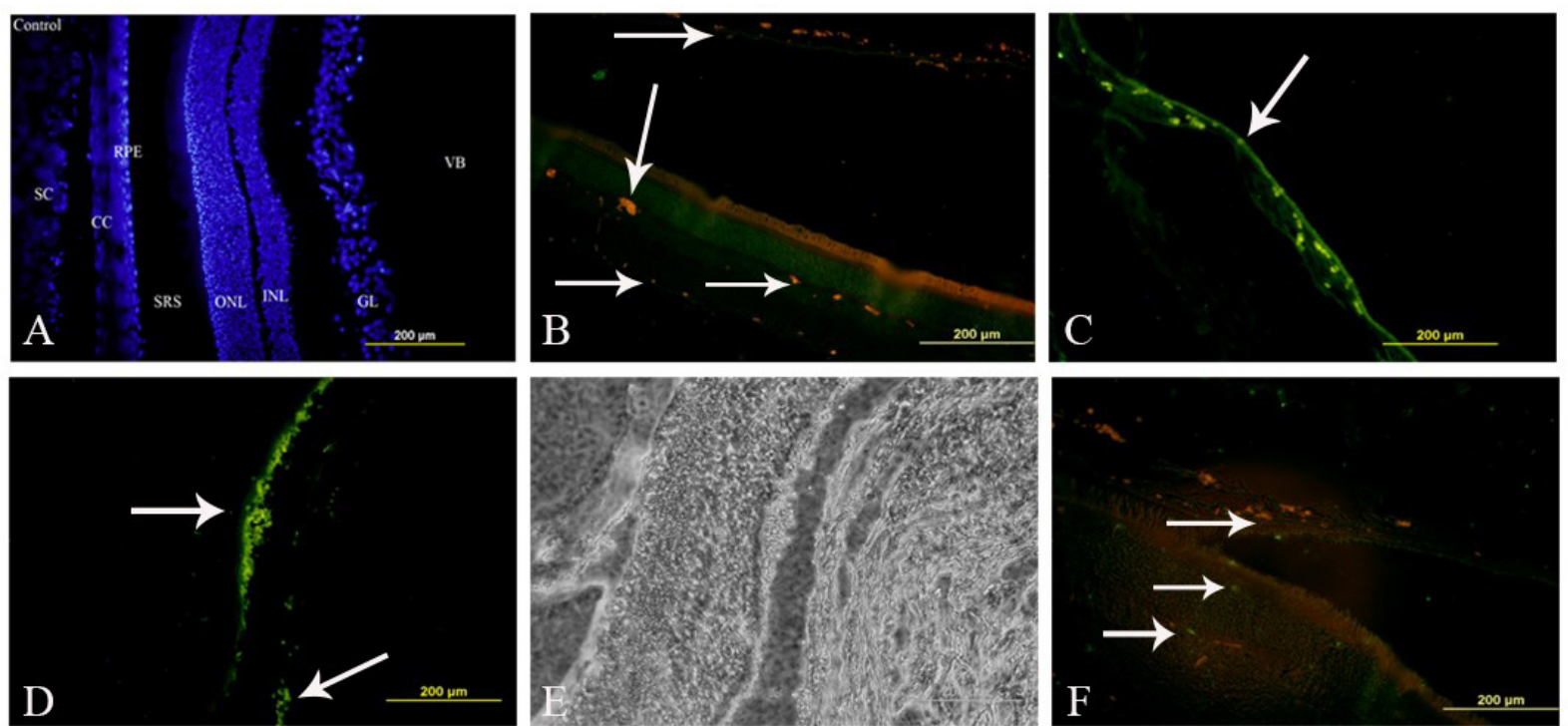

NEUR:SCIENCE

Figure 4. The immunohistochemical imaging sections prepared from the transplantation of neurospheres in sub-retinal space

A: The control group, the retina layers were stained with a DAPI nuclear marker; B: Cells transplanted in the RPE layer and the retinal layers (arrows); C-F: The accumulation of neurospheres in the RPE layer and the retinal layer after transplantation in the sub-retinal. The cells were observed both in the RPE layer and in the retinal layers. Magnification: $200 \mu \mathrm{m}$ for A-F, respectively.

SC: The sclera; CC: Choriocapillaris layer; RPE: Retinal Pigment Epithelium; ONL: Outer Nuclear Layer, INL: Inner Nuclear Layer; GL: Ganglionic Layer, SRS: Subretinal Space, VB: Vitreous Body. 
that sub-retinal space is a useful place for replacement therapy. There are several methods for the injection of cells into sub-retinal space (Qi et al., 2015). However, there are two approaches to inject the cell into the eye: Sub-retinal space, and Intravitreous injection (Dureau et al., 2000). In other words, sub-retinal transplantation results in surviving and more migratory cell transplantation. The sub-retinal space does not manifest the immune response to the implanted cells. In comparison, Intravitreous injection is less invasive and more general. However, studies indicated limitations for injection into vitreous space, like the internal limiting layer. There is a third way for stem cell injection that in this method tail vein is applied (Chung, Park, Ohn, Park, \& Hong, 2011; Johnson, Bull, \& Martin, 2010; Kollar, Cook, Atkinson, \& Brooke, 2009; Li et al., 2007).

Our evidence demonstrated that BMSCs derived neurosphere 7 days after transplantation conclusively expressed nuclear marker BrdU and were alive, might because of the secretion of cytokines from the degenerated RPE layer and the accumulation of BrdU positive neurosphere, remarkably similar study was established after transplantation of BMSCs in RPE degenerated applying sodium iodate (Klimanskaya et al., 2004). Two major destinations of our transplanted cells were the PRE layer and ONL, however, the ONL, INL, and the RPE are 3 common targets in cell replacement therapy (Tomita et al., 2002). A study indicated that the subretinal space is the final destination of transplanted BMSCs (Zhang \& Wang, 2010).

Studies reported that human embryonic stem cell transplantation into subretinal space in mice causes transformation into functional photoreceptors and improve photoreceptors light response also result in differentiation into RPE cells (Castro, Navajas, Farah, Maia, \& Rodrigues, 2013; Huang, Enzmann, \& Ildstad, 2011; Park et al., 2011). A study revealed that human embryonic stem cellsderived RPE transplantation survived for 1-4 weeks after transplantation in the sub-retina space and express RPEspecific markers (Castro et al., 2013). Nervous stem cells, like adult stem cells, can be incorporated with the PRE layer after transplantation and can be a place among them and form a new layer. The intravitreal transplantation of transformed nervous progenitor cells into neurosphere in mice results in migration, integration, and differentiation after 7 days (Castro et al., 2013).

\section{Conclusion}

Mesenchymal Stem Cells (MSCs), due to safety and convenience, are among the good choices for cell therapy. Neurosphere cells and neural stem cells, due to their em- bryonic proximity to retinal cells, can be a valuable source of cellular therapy for treating retinal diseases. These cells will not be rejected and can be applied in autologous transplantation. Here, we demonstrated that the BrdU positive BMSCs derived neurosphere transplantation into sub-retinal space could survive, migrate, and be integrated into the RPE layer and the ONL and INL layer. However, we encountered further limitations for the confirmation of neurosphere, determining the transplanted cells in IHC, and finally behavioral analysis, like electroretinography to suggest their effect on retinal cells activity.

\section{Ethical Considerations}

\section{Compliance with ethical guidelines}

There were no ethical considerations to be considered in this research.

\section{Funding}

This study was supported by the Research Center of Shefa Neuroscience at Khatam Alanbia Hospital, Tehran, Iran (Grant No. 86-N-105).

\section{Authors' contributions}

All authors equally contributed to preparing this article.

\section{Conflict of interest}

The authors declared no conflicts of interest.

\section{References}

Aboutaleb Kadkhodaeian, H., Tiraihi, T., Ahmadieh, H., Ziaei Ardakani, H., Daftarian, N., \& Taheri, T. (2017). Survival and migration of adipose-derived stem cells transplanted in the injured retina. Experimental and Clinical Transplantation, 16(2), 204-11. [DOI:10.6002/ect.2016.0235] [PMID]

Ambati, J., Ambati, B. K., Yoo, S. H., Ianchulev, S., \& Adamis, A. P. (2003). Age-related macular degeneration: Etiology, pathogenesis, and therapeutic strategies. Survey of Ophthalmology, 48(3), 257-93. [DOI:10.1016/S0039-6257(03)00030-4]

Castro, G., Navajas, E., Farah, M. E., Maia, M., \& Rodrigues, E. B (2013). Migration, integration, survival, and differentiation of stem cell-derived neural progenitors in the retina in a pharmacological model of retinal degeneration. International Scholarly Research Notices, 2013, 752161. [DOI:10.1155/2013/752161] [PMID] [PMCID]

Chen, M., Rajapakse, D., Fraczek, M., Luo, Ch., Forrester, J. V., \& $\mathrm{Xu}, \mathrm{H}$. (2016). Retinal pigment epithelial cell multinucleation in the aging eye - a mechanism to repair damage and main- 
tain homoeostasis. Aging Cell, 15(3), 436-45. [DOI:10.1111/ acel.12447] [PMID] [PMCID]

Chung, J. K., Park, T. K., Ohn, Y. H., Park, S. K., \& Hong, D. S (2011). Modulation of retinal wound healing by systemically administered bone marrow-derived mesenchymal stem cells. Korean Journal of Ophthalmology, 25(4), 268-74. [DOI:10.3341/ kjo.2011.25.4.268] [PMID] [PMCID]

Darabi, Sh., Tiraihi, T., Ruintan, A., Abbaszadeh, H. A., Delshad, A. R., \& Taheri, T. (2013). Polarized neural stem cells derived from adult bone marrow stromal cells develop a rosette-like structure. In Vitro Cellular \& Developmental Biology - Animal, 49(8), 638-52. [DOI:10.1007/s11626-013-9628-y] [PMID]

Dureau, P., Legat, L., Neuner-Jehle, M., Bonnel, S., Pecqueur, S., \& Abitbol, M., et al. (2000). Quantitative analysis of subretinal injections in the rat. Graefe's Archive for Clinical and Experimental Ophthalmology, 238(7), 608-14. [DOI:10.1007/ s004170000156] [PMID]

Enzmann, V., Row, B. W., Yamauchi, Y., Kheirandish, L., Gozal, D., \& Kaplan, H. J., et al. (2006). Behavioral and anatomical abnormalities in a sodium iodate-induced model of retinal pigment epithelium degeneration. Experimental Eye Research, 82(3), 441-8. [DOI:10.1016/j.exer.2005.08.002] [PMID]

Ferroni, L., Gardin, Ch., Tocco, I., Epis, R., Casadei, A., \& Vindigni, V., et al. (2012). In B. Weyand, M. Dominici, R. Hass, R. Jacobs \& C. Kasper (Eds.), Mesenchymal stem cells - basics and clinical application I. Advances in biochemical engineering/biotechnology (pp. 89-115). Vol. 129. Berlin/Heidelberg: Springer. [DOI:10.1007/10_2012_152] [PMID]

Franco, L. M., Zulliger, R., Wolf-Schnurrbusch, U. E. K., Katagiri, Y., Kaplan, H. J., \& Wolf, S., et al. (2009). Decreased visual function after patchy loss of retinal pigment epithelium induced by low-dose sodium iodate. Investigative Ophthalmology $\mathcal{E}$ Visual Science, 50(8), 4004-10. [DOI:10.1167/iovs.08-2898] [PMID]

Fu, L., Zhu, L., Huang, Y., Lee, T. D., Forman, S. J., \& Shih, C. C. (2008). Derivation of neural stem cells from mesenchymal stem cells: Evidence for a bipotential stem cell population. Stem Cells and Development, 17(6), 1109-22. [DOI:10.1089/ scd.2008.0068] [PMID] [PMCID]

Huang, Y., Enzmann, V., \& Ildstad, S. T. (2011). Stem cell-based therapeutic applications in retinal degenerative diseases. Stem Cell Reviews and Reports, 7(2), 434-45. [DOI:10.1007/s12015010-9192-8] [PMID] [PMCID]

Jiang, W., Zhang, W. Y., \& Chiou, G. C. Y. (2009). Effects of hydralazine on $\mathrm{NaIO}_{3}$-induced rat retinalpigment epithelium degeneration. International Journal of Ophthalmology, 2(2), 10612. http://www.ijo.cn/gjyken/ch/reader/view_abstract. aspx

Johnson, T. V., Bull, N. D., \& Martin, K. R. (2010). Identification of barriers to retinal engraftment of transplanted stem cells. Investigative Ophthalmology \& Visual Science, 51(2), 960-70. [DOI:10.1167/iovs.09-3884] [PMID] [PMCID]

Kaka, G. H. R., Tiraihi, T., Arab Kheradmand, J., \& Azizzadeh Delshad, A. R. (2009). A study on in-vitro transdifferentiation of rat bone marrow stromal cells into neuroepithelial-like cells. Iranian Red Crescent Medical Journal, 11(2), 133-9. https:/ / archive.ircmj.com/article/11/2/70925-pdf.pdf

Kadkhodaeian, H. A., Salati, A., \& Lashay, A. (2019). High efficient differentiation of human adipose-derived stem cells into retinal pigment epithelium-like cells in medium containing small molecules inducers with a simple method. Tissue and Cell, 56, 52-9. https://www.sciencedirect.com/science/article/abs/pii/S0040816618302842

Klimanskaya, I., Hipp, J., Rezai, K. A., West, M., Atala, A., \& Lanza, R. (2004). Derivation and comparative assessment of retinal pigment epithelium from human embryonic stem cells using transcriptomics. Cloning and Stem Cells, 6(3), 217-45. [DOI:10.1089/clo.2004.6.217] [PMID]

Koh, A. E. H., Alsaeedi, H. A., binti Abd Rashid, M., Lam, Ch. Harun, M. H. N., \& bin Mohd Saleh, M. F., et al. (2019). Retinal degeneration rat model: A study on the structural and functional changes in the retina following injection of sodium iodate. Journal of Photochemistry and Photobiology B: Biology, 196, 111514. [DOI:10.1016/j.jphotobiol.2019.111514] [PMID]

Kollar, K., Cook, M. M., Atkinson, K., \& Brooke, G. (2009). Molecular mechanisms involved in mesenchymal stem cell migration to the site of acute myocardial infarction. International Journal of Cell Biology, 2009, 904682. [DOI:10.1155/2009/904682] [PMID] [PMCID]

Lee, E., \& MacLaren, R. E. (2011). Sources of Retinal Pigment Epithelium (RPE) for replacement therapy. British Journal of Ophthalmology, 95(4), 445-9. [DOI:10.1136/bjo.2009.171918] [PMID]

Li, X., Liao, D., Gong, P., Yuan, Q., \& Tan, Zh. (2009). Neural differentiation of adipose-derived stem cells by indirect co-culture with Schwann cells. Archives of Biological Sciences, 61(4), 703-11. [DOI:10.2298/ABS0904703L]

Li, Y., Atmaca-Sonmez, P., Schanie, C. L., Ildstad, S. T., Kaplan H. J., \& Enzmann, V. (2007). Endogenous bone marrowderived cells express retinal pigment epithelium cell mark ers and migrate to focal areas of RPE damage. Investigative Ophthalmology \& Visual Science, 48(9), 4321-7. [DOI:10.1167/ iovs.06-1015] [PMID]

Liu, Y., Li, Y., Wang, Ch., Zhang, Y., \& Su, G. (2019). Morphologic and histopathologic change of sodium iodate-induced retinal degeneration in adult rats. International Journal of Clinical and Experimental Pathology, 12(2), 443-54. [PMID] [PMCID]

Liu, Y., Liu, L., Ma, X., Yin, Y., Tang, B., \& Li, Z. (2013). Characteristics and neural-like differentiation of mesenchymal stem cells derived from foetal porcine bone marrow. Bioscience Reports, 33(2), e00032. [DOI:10.1042/BSR20120023] [PMID]

Machalińska, A., Lubiński, W., Kłos, P., Kawa, M., Baumert, B., \& Penkala, K., et al. (2010). Sodium iodate selectively injuries the posterior pole of the retina in a dose-dependent manner: Morphological and electrophysiological study. Neurochemical Research, 35(11), 1819-27. [DOI:10.1007/s11064-010-0248-6] [PMID] [PMCID]

Mead, B., Berry, M., Logan, A., Scott, R. A. H., Leadbeater, W., \& Scheven, B. A. (2015). Stem cell treatment of degenerative eye disease. Stem Cell Research, 14(3), 243-57. [DOI:10.1016/j. scr.2015.02.003] [PMID] [PMCID]

Naghdi, M., Tiraihi, T., Mesbah-Namin, S. A. R., \& Arabkheradmand, J. (2009). Induction of bone marrow stromal cells into cholinergic-like cells by nerve growth factor. Iranian Biomedical Journal, 13(2), 117-23. [PMID]

Parameswaran, S., \& Krishnakumar, S. (2017). Pluripotent stem cells: A therapeutic source for age-related macular de- 
generation. Indian Journal of Ophthalmology, 65(3), 177-83. [DOI:10.4103/ijo.IJO_1026_15] [PMID] [PMCID]

Park, S. S., Moisseiev, E., Bauer, G., Anderson, J. D., Grant, M. B., \& Zam, A., et al. (2017). Advances in bone marrow stem cell therapy for retinal dysfunction. Progress in Retinal and Eye Research, 56, 148-65. [DOI:10.1016/j.preteyeres.2016.10.002] [PMID] [PMCID]

Park, U. C., Cho, M. S., Park, J. H., Kim, S. J., Ku, S. Y., \& Choi, Y. M., et al. (2011). Subretinal transplantation of putative retinal pigment epithelial cells derived from human embryonic stem cells in rat retinal degeneration model. Clinical and $E x-$ perimental Reproductive Medicine, 38(4), 216-21. [DOI:10.5653/ cerm.2011.38.4.216] [PMID] [PMCID]

Paulus, T. V. M., \& de Jong, M. D. (2006). Age-related macular degeneration. The New England Journal of Medicine, 355(14) 1474-85. [DOI:10.1056/NEJMra062326] [PMID]

Pennesi, M. E., Neuringer, M., \& Courtney, R. J. (2012). Animal models of age related macular degeneration. Molecular Aspects of Medicine, 33(4), 487-509. [DOI:10.1016/j.mam.2012.06.003] [PMID] [PMCID]

Qi, Y., Dai, X., Zhang, H., He, Y., Zhang, Y., \& Han, J., et al (2015). Trans-corneal subretinal injection in mice and its effect on the function and morphology of the retina. PloS One, 10(8), e0136523. [DOI:10.1371/journal.pone.0136523] [PMID]

Schwartz, S. D., Nagiel, A., \& Lanza, R., editors. (2017). Cellular therapies for retinal disease: A strategic approach. Cham: Springer. [DOI:10.1007/978-3-319-49479-1]

Song, C. G., Zhang, Y. Z., Wu, H. N., Cao, X. L., Guo, C. J., \& Li, Y. Q., et al. (2018). Stem cells: A promising candidate to treat neurological disorders. Neural Regeneration Research, 13(7), 1294-304. [DOI:10.4103/1673-5374.235085] [PMID] [PMCID]

Stanga, P. E., Kychenthal, A., Fitzke, F. W., Halfyard, A. S. Chan, R., \& Bird, A. C., et al. (2002). Retinal pigment epithelium translocation after choroidal neovascular membrane removal in age-related macular degeneration. Ophthalmology, 109(8), 1492-8. https:/ / www.sciencedirect.com/science/article/abs/pii/S0161642002010990

Tomita, M., Adachi, Y., Yamada, H., Takahashi, K., Kiuchi, K., \& Oyaizu, H., et al. (2002). Bone marrow-derived stem cells can differentiate into retinal cells in injured rat retina. Stem Cells, 20(4), 279-83. [DOI:10.1634/stemcells.20-4-279] [PMID]

Trounson, A., \& McDonald, C. (2015). Stem cell therapies in clinical trials: Progress and challenges. Cell Stem Cell, 17(1), 11-22. [DOI:10.1016/j.stem.2015.06.007] [PMID]

Weed, L. S., \& Mills, J. A. (2017). Strategies for retinal cell generation from human pluripotent stem cells. Stem Cell Investigation, 4(7), 65. [DOI:10.21037/sci.2017.07.02] [PMID] [PMCID]

Wu, S., Suzuki, Y., Ejiri, Y., Noda, T., Bai, H., \& Kitada, M., et al. (2003). Bone marrow stromal cells enhance differentiation of cocultured neurosphere cells and promote regeneration of injured spinal cord. Journal of Neuroscience Research, 72(3), 34351. [DOI:10.1002/jnr.10587] [PMID]

Yang, J., Yan, Y., Ciric, B., Yu, Sh., Guan, Y., \& Xu, H., et al. (2010). Evaluation of bone marrow-and brain-derived neural stem cells in therapy of central nervous system autoimmunity. The American Journal of Pathology, 177(4), 1989-2001. [DOI:10.2353/ajpath.2010.091203] [PMID] [PMCID]
Yu, K., Ge, J., Summers, J. B., Li, F., Liu, X., \& Ma, P., et al. (2008) TSP-1 secreted by bone marrow stromal cells contributes to retinal ganglion cell neurite outgrowth and survival. PloS One, 3(6), e2470. [DOI:10.1371/journal.pone.0002470] [PMID]

Zhang, Y., \& Wang, W. (2010). Effects of bone marrow mesenchymal stem cell transplantation on light-damaged retina. Investigative Ophthalmology \& Visual Science, 51(7), 3742-8. [DOI:10.1167/iovs.08-3314] [PMID]

Zieger, M., \& Punzo, C. (2016). Improved cell metabolism prolongs photoreceptor survival upon retinal-pigmented epithelium loss in the sodium iodate induced model of geographic atrophy. Oncotarget, 7(9), 9620-33. [DOI:10.18632/oncotarget.7330] [PMID] [PMCID] 